\title{
La contribution de l'activité pétrolière à la connaissance des nappes
}

\author{
par Georges Blanc \\ de I'Institut Français du Pétrole, Pau \\ et André Lagarde \\ du BEICIP-Franlab, Sophia-Antipolis
}

\section{INTRODUCTION}

La contribution de l'activité pétrolière à la connaissance des nappes est envisagée sous plusieurs aspects :

- L'utilisation des informations acquises lors de l'exploration pétrolière provenant de la géophysique, de la géologie, du forage, des diagraphies et des essais de puits.

- La réutilisation des puits pétroliers à des fins de surveillance ou d'exploitation des nappes profondes.

- L'application à l'évaluation des nappes des techniques développées par l'industrie pétrolière pour la gestion des gisements pétroliers et pour l'interprétation des mesures dans les puits (diagraphies et essais de puits par exemple).

Quelques exemples illustrent chacun de ces trois aspects.

\section{1 UTILISATION DES INFORMATIONS ACQUISES LORS DE L'EXPLORATION PÉTROLIÈRE}

Dans certaines contrées désertiques, l'étude des nappes profondes et des ensembles hydrogéologiques ne peut s'appuyer que sur les données de l'exploration pétrolière. Il n'est en effet pas question de lancer une campagne de forages et de mesures dans ce seul but.

Un excellent exemple d'utilisation des données ainsi acquises est illustré dans les études des ressources en eau de la Libye menées en 1969 par J.R. Jones [1] et dans les études menées entre cette date et 1977 , et décrites par P. Pallas [2]. Ces études qui concernent les cinq grands systèmes hydrogéologiques libyens sont basées sur des sections géologiques contrôlées par des puits pétroliers. Les informations recueillies au cours des forages ont été ains mises à profit pour la construction des coupes lithologiques et pour l'étude de la qualité des eaux des aquifères traversés.

Un autre exemple concerne l'étude des ressources en eau du Sahara septentrional menée par l'UNESCO de 1968 à 1972 et pour laquelle les informations recueillies lors de l'exploration pétrolière de cette zone ont été utilisées. Le rapport de l'UNESCO [3] mentionne en particulier que pour cette étude, « l'utilisation des résultats de l'exploration géophysique s'est révélée extrêmement utile pour définir de façon plus précise les caractéristiques géométriques des réservoirs et principalement du réservoir profond $»$ Les côtes du toit et du mur des aquifères traversés par 615 forages profonds ont également été utilisées pour cette définition géométrique. L'étude des diagraphies acquises sur 183 forages a permis d'évaluer la porosité moyenne des nappes, leur hauteur utile ainsi que la salinité de leurs eaux.

\section{RÉUTILISATION DE PUITS PÉTRO- LIERS}

Dans certaines régions, les puits utilisés lors de l'exploitation pétrolière ont pu être réutilisés à des fins d'étude et de surveillance des nappes.

Dans les études des ressources en eau de la Libye citées précédemment, les puits de moyenne profondeur forés par les compagnies pétrolières pour leur besoin en eau durant la phase d'exploration, et laissés dans l'état, ont permis d'organiser un contrôle de la qualité des eaux et une surveillance piézométrique des nappes concernées. 


\section{APPLICATION DES TECHNIQUES D'ÉVALUATION DES GISEMENTS PÉTROLIERS}

\subsection{Evaluation des nappes}

La similitude des problèmes posés par la gestion des nappes et par la gestion des réservoirs pétroliers a permis à l'industrie pétrolière de mettre, dès le début des années 60 , les outils de simulation analogique et numérique des écoulements en milieux poreux à la disposition des hydrogéologues. Ceci explique pourquoi deux sociétés issues du milieu pétrolier (Franlab pour l'Institut Français du Pétrole et Géohydraulique pour Géopétrole) ont été parmi les premières sociétés de consultants à fournir des services dans ce domaine.

Un article de A. Lagarde [4] fait état, en 1963, des tous premiers travaux de simulations de nappes à l'aide d'un réseau analogique résistance-capacité utilisé à l'Institut Français du Pétrole comme simulateur de gisements. Par la suite, les simulateurs numériques de nappes issus des outils développés pour les besoins des études de gisements ont été adaptés et utilisés dans de nombreuses études de gestion des ressources en eau. A. Lagarde et $M$. Lanchon [5] décrivent dès 1966 un exemple d'utilisation d'un simulateur de gisement pour les études de régimes permanents et transitoires des nappes. En 1973, les méthodes numériques basées sur les éléments finis étaient utilisées pour l'étude des écoulements dans les digues et barrages en terre permettant une meilleure adéquation entre la forme du domaine simulé et les techniques de maillage ( $G$. Blanc, Y. Baradat et A. Lagarde [6]). Dans les années 80, l'utilisation des microordinateurs, dans le cadre du projet UNDP RAB 80/011, a redonné un essor à la simulation des nappes aquifères à des fins de gestion des ressources en eau, en permettant la diffusion des simulateurs sur des ordinateurs plus facilement accessibles par les hydrogéologues (P. Pallas et G. Blanc [7]).

Plus récemment, les recherches de l'industrie pétrolière en matière d'écoulements multiphasiques ont été mises à profit pour engager un projet de modélisation numérique de la pollution des sols et des eaux par les produits organiques et pour la simulation des procédés de dépollution des nappes. Ces travaux menés dans le cadre du projet EUREKA RESCOPP par l'Institut Français du Pétrole, BURGEAP et des sociétés italiennes du groupe pétrolier ENI, doivent donner naissance au logiciel SIMUSCOPP.

\subsection{Interprétation des mesures dans les puits}

Les problèmes auxquels l'hydrogéologue et l'ingénieur de réservoir ont à faire face dans ce domaine sont similaires et les transferts technologiques sont fructueux.

Pour ce qui concerne l'interprétation des diagraphies, les travaux menés dans le cadre de l'étude UNESCO [3] ont montré tout l'intérêt de recourir à l'expérience pétrolière dans ce domaine afin de quantifier les volumes en place ainsi que la salinité des eaux.

Dans le domaine de l'interprétation des essais de puits, les résultats acquis ces dernières années en matière d'utilisation des dérivées des courbes de pression par D. Tiab et $A$. Kumar [8] ont conduit à une amélioration de l'analyse de ces essais. Les courbes dérivées permettent en effet d'identi- fier les modèles d'interprétation adaptés à la situation hydrogéologique, de quantifier les effets de capacité du puits ainsi que l'endommagement du trou par le forage, ou bien son éventuelle amélioration par les techniques de stimulation (fracturation artificielle ou acidification).

\section{CONCLUSIONS}

Le présent article ne fait état que de la contribution de l'activité pétrolière à la connaissance des nappes. En fait les transferts technologiques sont bilatéraux dans cette branche des géosciences qu'est l'hydraulique souterraine appliquée à l'eau ou aux hydrocarbures. L'article de T. Ghalem et A. Lagarde [9] concerne l'utilisation des ressources en eau du Sahara pour les besoins de l'exploitation pétrolière. Il constitue un exemple de retour vers l'industrie pétrolière de résultats obtenus par une étude hydrogéologique elle même basée sur les apports de cette même industrie.

Dans le futur, ces échanges ne feront que s'amplifier, et sont déjà conduits par une préoccupation commune en matière de traitement des problèmes liés à la préservation de l'environnement

\section{Références}

[1] JONES J.R. (1969). — « Groundwater in Libya. A summary of hydrogeology of the southern Mediterranean littoral of the north central Sahara », L.A.R. USGS. Open-File Rep.

[2] Pallas P. (1977), - «Water Resources of the Socialist Peoples's Libyan Arab Jamahiriya ", in « The Geology of Libya Proceedings of the Second Symposium of the Geology of Libya », London Academic Press 1980.

[3] UNESCO. «Nappe du Continental Intercalaire. Rapport du projet REG 100 établi par l'UNESCO, plaquette $2 \%$. Paris, avril 1992

[4] LaGARDE (A.). - «Un exemple de résolution analogique à l'aide d'un réseau R.C. d'un problème de diffusivité dans une nappe aquifère ». Mémoires et travaux de la S.H.F., numéro II, 1973.

[5] Lagarde A. et LANCHON M. - "Simulation par modèle mathématique des écoulements souterrains permanents et transitoires. Application à l'étude de l'exploitation des nappes $\%$. Comité Technique n ${ }^{\circ} 81$ de la S.H.F., 17 et 18 novembre 1966.

[6] Blanc G., Baradat Y. et Lagarde A. - «Utilisation de modèles mathématiques multidimensionnels pour l'étude des écoulements dans les digues et barrages en terre $»$. La Houille Blanche, $n^{\circ} 5 / 6,1973$.

[7] Pallas G. et Blanc G. - "L'eau et le Maghreb. Gestion des eaux souterraines ». 1988.

[8] TIAB D. et Kumar A. - «Application of the $\mathrm{p}_{\mathrm{D}}$ Function to Interference Analysis » J. Pet. Tech. (august 1980), 1701-1708.

[9] Lagarde. A., Ghalem T. et Delhomme A. - « Water for oil A Rational and Regional Approach of Water Supply for Secondary Recovery in Hassi-Messaoud Field, Algeria ». Paper SPE 9317 presented at the 55th Annual Fall Conference and Exhibition of the Society of Petroleum Engineers. Dallas, September 1980. 